\title{
'True' Chirality Transfer from Silicon to Carbon
}<smiles></smiles>

$85 \%$ ee $\underset{\left[(\text { phen }) \mathrm{Pd}(\mathrm{Me})\left(\mathrm{OEt}_{2}\right)\right]^{+}\left[\mathrm{BAr}_{4}\right]^{-}(5 \mathrm{~mol} \%)}{\mathrm{CH}_{2} \mathrm{Cl}_{2},-55^{\circ} \mathrm{C}}$

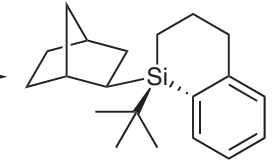

$99 \%$ chirality transfer $58 \%$ yield exo:endo $>99: 1$

chiral amplification
Metal-Catalyzed

Asymmetric

Synthesis and

Stereoselective

Reactions

Key Words

chirality transfer asymmetric amplification hydrosilation
Significance: Palladium-catalyzed hydrosilation giving perfect stereoselectivity has been accomplished. A chirality transfer from silicon to carbon resulting in asymmetric amplification and highly optical pure products is the result. A positive nonlinear effect has implied a matched mismatched scenario involved in the proposed mechanism.
Comment: 'True' chirality transfer from silicon to carbon has long been an elusive transformation in chemistry. Not only does this paper describe this transformation giving high ee's, but also their protocol involves a somewhat rare case of asymmetric amplification. The origin of this very interesting asymmetric amplification still awaits further investigation. 